\title{
Preparation of Highly Perfect Aluminum Crystal by Cold-Crucible Induction Melting in Ultra-high Vacuum *
}

\author{
Hiroto Osono, Hiroshi Maeta, Kikuo Matsusaka and Takao Kino \\ Faculty of Engineering, Hiroshima Kokusai Gakuin University, Hiroshima 739-0321, Japan
}

\begin{abstract}
To make a highly perfect aluminum crystal, high purity aluminum was melted in ultra-high vacuum of $2 \times 10^{-7} \mathrm{~Pa}$ for $2 \mathrm{~h}$ using a coldcrucible induction furnace. The surface of as-melted ingot had a metallic luster, and heavy oxidization on the surface was not recognized. In the ingot, a large cylindrical-single crystal of diameter $60 \mathrm{~mm}$ and length $40 \mathrm{~mm}$ grew and dislocation density in the crystal was about $10^{7} \mathrm{~m}^{-2}$. The purity of the refined aluminum was estimated to be near $99.9999 \%$ from the residual resistance ratio. The impurity elements removed with high purification efficiency had a distribution coefficient of less than unit. A large amount of highly perfect aluminum crystal of ultra-high purity was produced by melting only once under the ultra-high vacuum.
\end{abstract}

(Received July 23, 2001; Accepted November 15, 2001)

Keywords: ultra-high purity aluminum, large single crystal, low dislocation density, highly perfect aluminum crystal, ultra-high vacuum melting, cold-crucible induction furnace, glow discharge mass spectrometry, distribution coefficient

\section{Introduction}

Purification of aluminum has been performed mainly by zone melting method. The highest purity aluminum reported hitherto was obtained by a combination of zone melting and the cropping procedure, in which impurity-enriched regions in both top and tail sides of the zone melted rod were separated after each zone pass. ${ }^{1)}$ The residual resistance ratio $R(300 \mathrm{~K}) / R(4.2 \mathrm{~K})$ was more than $1.5 \times 10^{5}$ in the bulk value. ${ }^{2)}$ Using such a high purity aluminum, appearance of ballistic effect of conduction electrons ${ }^{3)}$ or slowing down of generation rate of thermal vacancy ${ }^{4)}$ etc. ${ }^{5)}$ have been reported. Therefore the development of preparation method of such a highly perfect crystal is demanded. However, zone melting method takes several ten hours for one zone pass, and furthermore it is necessary to repeat the zone pass.

The purpose of the present work is to investigate how effective the melting under ultra-high vacuum is in the purification of aluminum and obtaining highly perfect aluminum crystal.

\section{Experimental Procedures}

The material used in the present work was $99.999 \%$ aluminum produced by Sumitomo Chemical Co. Ltd. The sample was a cylinder $60 \mathrm{~mm}$ in diameter and $100 \mathrm{~mm}$ in length. In order to avoid the contamination from the surface during the melting, the sample surface was electro-polished in a bath of 1 part hydrochloric acid-4parts ethyl alcohol, and then was rinsed in distilled water, ethyl alcohol and acetone in the order.

The sample was melted in a cold-crucible of inner diameter $70 \mathrm{~mm}$ and depth $130 \mathrm{~mm}$, using an ultra-high vacuummelting furnace made by ULVAC-PHI. Inc. The best vacuum achieved in the apparatus was $2.7 \times 10^{-8} \mathrm{~Pa}$, and it dropped to $4 \times 10^{-6} \mathrm{~Pa}$ immediately after the sample melting, but the vacuum recovered to $2 \times 10^{-7} \mathrm{~Pa}$ within several minutes. The melting was performed for 2 hours by induction heating un-

*This Paper was Originally Published in J. Japan Inst. Metals 65 (2001) $143-146$. der the following conditions: $22 \mathrm{kHz}, 570 \mathrm{~V}$ and $78 \mathrm{~kW}$. In the cooling process, the induction power was decreased gradually for 10 minutes.

After the vacuum melting, the sample was cut to plates $3 \mathrm{~mm}$ in thickness using a spark cutter. These samples were etched chemically in mixture aqua regia and hydrogen fluoride, and then the surface was observed to know the grain size and the dislocation densities. The dislocation density was estimated from the etch pits using a scanning electron microscope. Several samples were annealed cyclically between $170^{\circ} \mathrm{C}$ and $270^{\circ} \mathrm{C}$ six times in vacuum of $1 \times 10^{-4} \mathrm{~Pa}$. Several parts of these plates were cut to stick like specimen of cross section $3 \times 3 \mathrm{~mm}^{2}$ and length $35 \mathrm{~mm}$ for the electrical resistance measurement.

Residual impurities in the starting material and the purified sample were analyzed by a method of glow discharge mass spectrometry (GDMS) for 42 elements.

\section{Experimental Results and Discussion}

\subsection{Surface treatment of samples}

The depth profiles of elements near the sample surfaces were examined using a glow discharge optical emission spectrometer (GDOES), after the surface was electro-polished and rinsed by acetone. The results are shown in Fig. 1(a), and results of similar measurement for the surface just after the spark cutting are seen in Fig. 1(b) for comparison. The ordinate is the emission intensity and the abscissa is the etching time by argon ions, which is related to the depth from the initial surface of the sample, in the relation $50 \mathrm{~nm} \mathrm{~s}^{-1}$ in the etching rate. The emission intensity of aluminum is about 5 in the scale after $0.5 \mathrm{~s}$ in the etching time. Thus the part of $30 \mathrm{~nm}$ under the surface is considered to be the matrix material in the electro-polished sample. Furthermore, the composite layer containing carbon and sulfur is about $10 \mathrm{~nm}$. On the other hand, in the spark cut sample, the emission intensity of aluminum is about 3 even at the etching time $6 \mathrm{~s}$. Therefore the electro-polishing and rinse by acetone is necessary to eliminate the surface contamination. 

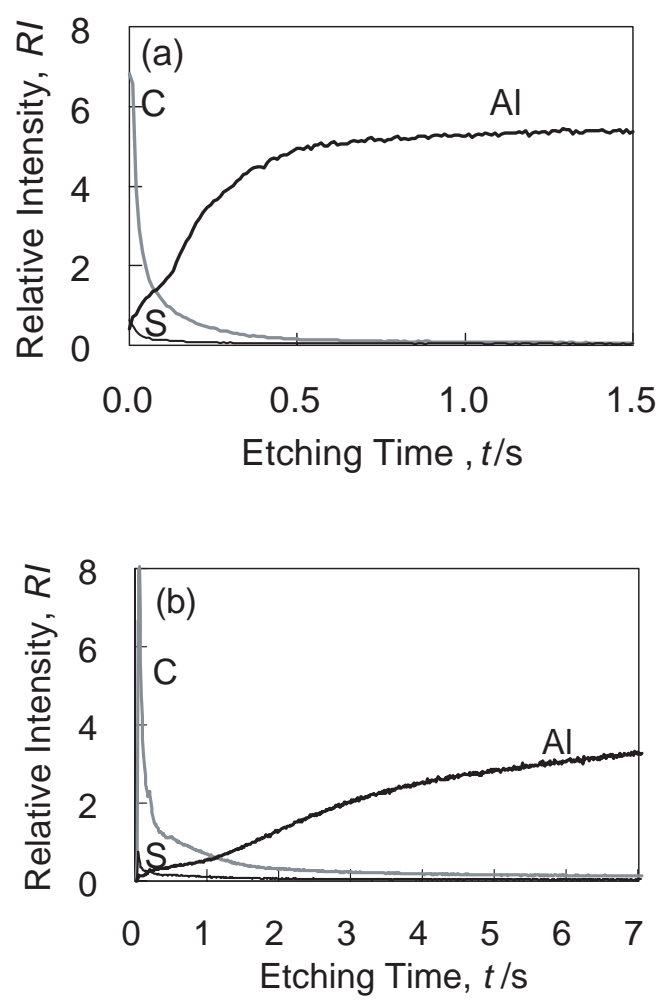

Fig. 1 Depth profiles of elements near the sample surface of the cleaned sample (a) and the spark-cutting sample (b).

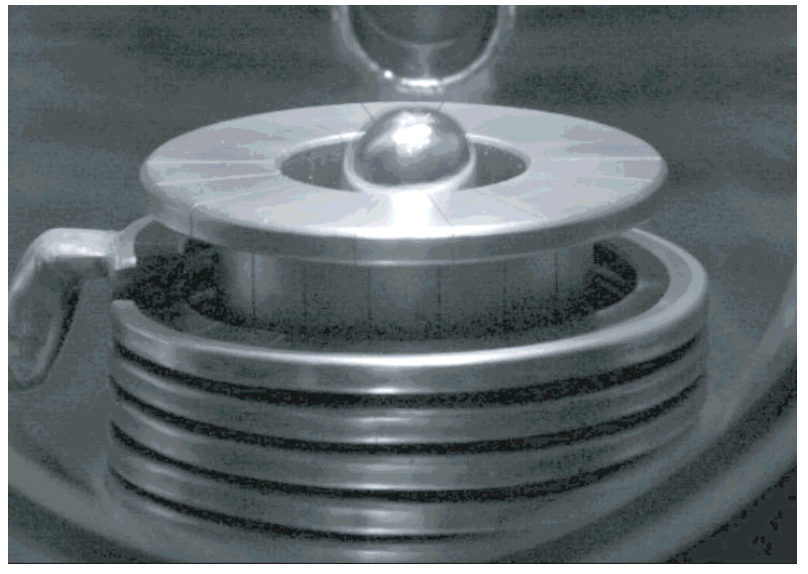

Fig. 2 The melting state of aluminum sample in the cold-crucible.

\subsection{Melting state of sample}

Figure 2 shows the melting state of aluminum sample in a cold-crucible. The top of the sample thrust out from the crucible; thus the melting sample seems to be out of contact with the wall of the cold-crucible. The sample was taken out from the crucible after the vacuum melting for $2 \mathrm{~h}$ and the side surface is shown in Fig. 3(a). The surface has a metallic luster, and this luster comes from the melting in ultra-high vacuum and the adequate treatment of sample surface.

\subsection{Crystal grain and dislocation density}

The ingot was cut to two parts along the cylindrical axis, and the surface was etched by mixture aqua regia and hydrogen fluoride in order to show the grain structure as seen in Fig. 3(b). The grain size in the bottom part of the ingot is several millimeters, but large grains are observed in the other
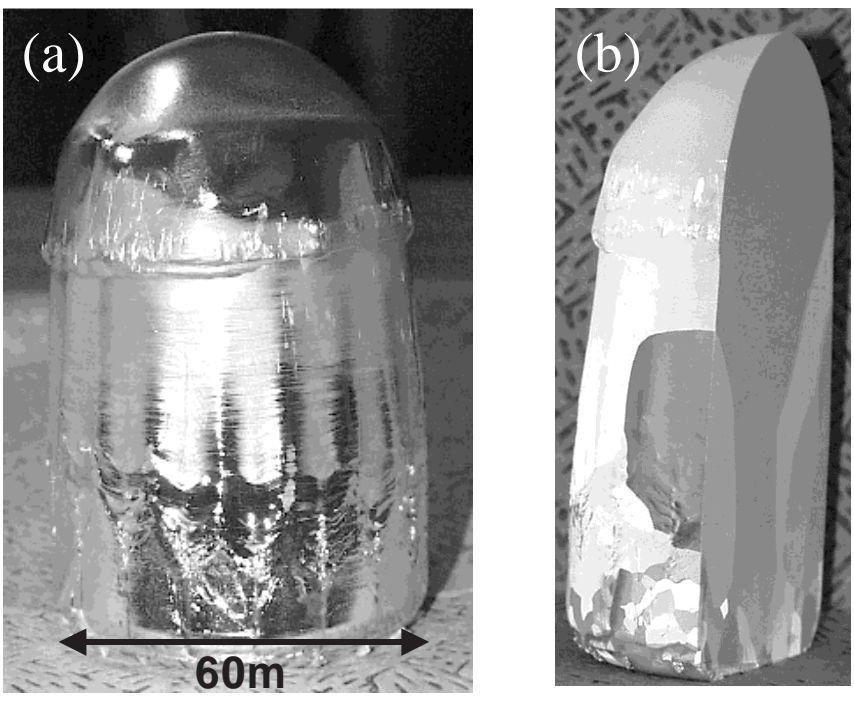

Fig. 3 Photographs of the aluminum ingot (a) and the etched surface after cutting the sample, which shows grain structure (b).

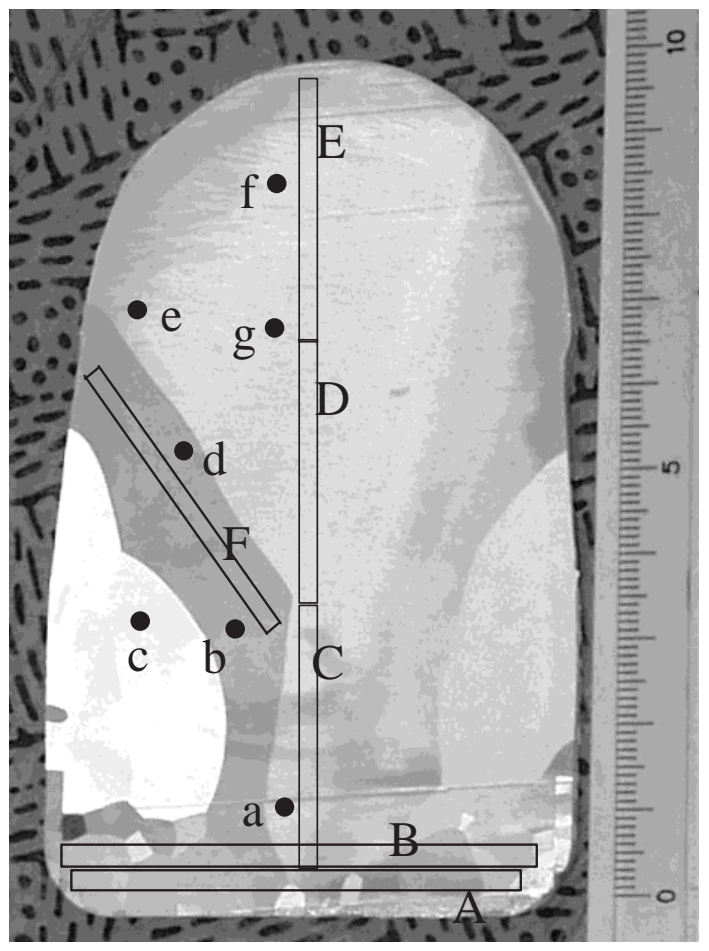

Fig. 4 Sampling locations for the observation of etch pits and the measurement of the electrical resistance. Small letters in the figure show the spots where the dislocation density was estimated and large letters show the samples for resistance measurement.

part. In particular, the upper part of the ingot converted to a large cylindrical single crystal $60 \mathrm{~mm}$ in diameter and $40 \mathrm{~mm}$ in length. Such a crystal growth is considered to come from the improvement of sample purity and slow cooling rate during the solidification after the melting.

Spots where the dislocation densities have been estimated are shown by small letters in Fig. 4, and the densities are tabulated in Table 1. The dislocation density increases from the bottom part to the upper part, and the central part has the maximum density. These results can be explained by the solidification process that start from the bottom part to the side 
Table 1 Dislocation density at the spots shown in Fig. 4.

\begin{tabular}{ccc}
\hline \multirow{2}{*}{ Spot } & \multicolumn{2}{c}{ Dislocation density, $D / \mathrm{m}^{-2}$} \\
\cline { 2 - 3 } & As melted & After cyclic-annealing \\
\hline a & $6 \times 10^{6}$ & $6 \times 10^{6}$ \\
b & $1 \times 10^{7}$ & $1 \times 10^{7}$ \\
c & $5 \times 10^{7}$ & $2 \times 10^{7}$ \\
d & $5 \times 10^{7}$ & $2 \times 10^{7}$ \\
e & $6 \times 10^{7}$ & $2 \times 10^{7}$ \\
f & $5 \times 10^{8}$ & $3 \times 10^{7}$ \\
g & $8 \times 10^{8}$ & $4 \times 10^{7}$ \\
\hline
\end{tabular}

Table 2 Residual resistance ratio $(R R R)$ of the sample marked by rectangle in Fig. 4.

\begin{tabular}{ccccccc}
\hline Sample & A & B & C & D & E & F \\
$R R R$ & $6 \times 10^{3}$ & $7 \times 10^{3}$ & $9.4 \times 10^{3}$ & $1.3 \times 10^{4}$ & $1.3 \times 10^{4}$ & $1.3 \times 10^{4}$ \\
\hline
\end{tabular}

Table 3 Chemical composition analyzed by GDMS method.

\begin{tabular}{ccc}
\hline & \multicolumn{2}{c}{ Chemical composition, } \\
Element & \multicolumn{2}{c}{ C/mass-ppm } \\
\cline { 2 - 3 } & Starting material & After melting \\
\hline $\mathrm{B}$ & 0.11 & 0.010 \\
$\mathrm{Mg}$ & 0.89 & $<0.001$ \\
$\mathrm{Si}$ & 1.8 & 0.74 \\
$\mathrm{Ti}$ & 0.22 & 0.21 \\
$\mathrm{~V}$ & 0.11 & 0.11 \\
$\mathrm{Cr}$ & 0.060 & 0.058 \\
$\mathrm{Mn}$ & 0.006 & 0.006 \\
$\mathrm{Fe}$ & 0.62 & 0.15 \\
$\mathrm{Ni}$ & 0.41 & 0.022 \\
$\mathrm{Cu}$ & 0.63 & 0.45 \\
$\mathrm{Zn}$ & 0.028 & $<0.001$ \\
$\mathrm{Zr}$ & 0.005 & 0.005 \\
$\mathrm{Sb}$ & 0.001 & $<0.001$ \\
$\mathrm{La}$ & 0.17 & $<0.001$ \\
$\mathrm{Ce}$ & 0.45 & $<0.001$ \\
$\mathrm{Nd}$ & 0.087 & $<0.001$ \\
$\mathrm{Th}$ & 0.054 & $<0.0003$ \\
$\mathrm{U}$ & 0.04 & $<0.0003$ \\
\hline $\mathrm{Total}$ & 5.691 & 1.767 \\
\hline
\end{tabular}

surface and finally to the central part of the ingot. The dislocation density decreased furthermore by the cyclic annealing as seen in Table 1. In the central part, the dislocation density decreased to $1 / 20$ by the cyclic annealing. The dislocation density after the cyclic annealing is $10^{7} \mathrm{~m}^{-2}$ over the almost whole part of the ingot except the bottom part.

\subsection{Estimation of purity}

The purity of samples was estimated by the measurement of the residual resistance ratio $R(300 \mathrm{~K}) / R(4.2 \mathrm{~K})$. Specimens for the electrical resistance measurement are denoted by rectangles with capital letters A-E in Fig. 4, and the residual resistance ratio are tabulated in Table 2 . The resistance ratio of the starting material is $8 \times 10^{3}$. Specimens $\mathrm{A}$ and $\mathrm{B}$ are within several millimeters from the bottom, and possibly contacts to the cold-crucible from the beginning during the solidification process. Therefore the resistance ratio in this region of $6-7 \times 10^{3}$ suggests a contamination from the crucible. The ratio of specimen $C$ which is lower part of the ingot is not less than that of the starting material. Specimens D, E and $\mathrm{F}$ are central or upper parts of the ingot, and their ratio are $1.3 \times 10^{4}$. Therefore the ratio of ingot is almost $1.3 \times 10^{4}$ over $80 \%$ of the ingot except the bottom part $20 \mathrm{~mm}$ in thickness. This result consists with the dislocation density after the cyclic annealing as seen in Table 1.

Another starting material with the residual resistance ratio $1.3 \times 10^{4}$ were melted in the same manner, and the residual resistance ratio after the vacuum melting was improved to $1.9 \times 10^{4}$. From this fact, we can expect the further improvement of the purity by repeating the melting under the ultra-high vacuum.

\subsection{Analysis of residual impurities}

The results analyzed by the GDMS method are tabulated in Table 3 for materials before and after the vacuum melting. According to the results, 18 elements contain more than 0.001 mass-ppm in the starting material, and the total concentration of the residual impurity is about 6 mass-ppm. On the other hand, in the material after melting, the total concentration is less than 2 mass-ppm. In order to compare this chemical analysis with the measured residual resistance ratio, the residual resistivity is estimated using the specific electrical resistivity ${ }^{6-8)}$ of various impurities in aluminum. The results are tabulated for both materials in Table 4 . Thus the estimated residual resistivity is 5.31 and $2.42 \mathrm{p} \Omega \cdot \mathrm{m}$ for materials before and after melting, respectively. The residual resistance ratio estimated from the above values, using $27.33 \mathrm{n} \Omega \mathrm{m}$ as the electrical resistivity at $300 \mathrm{~K}$, is $5.1 \times 10^{3}$ and $1.13 \times 10^{4}$, respectively. These values are almost the same as the measured residual resistance ratio described in the last paragraph.

In order to see the efficiency of purification for each impurity element, the amount of decrease in the impurity concentration during the vacuum melting is divided by the initial concentration, and this value will be defined as the purification efficiency herein. The results are tabulated in Table 5 together with the melting point, the vapor pressure at $660^{\circ} \mathrm{C}$ and the distribution coefficient ${ }^{9}$ ) for each element in aluminum. It is clear that elements with high purification efficiency have a distribution coefficient of less than unit except the element of boron. However, no correlation is found between the efficiency and the melting point or the vapor pressure.

\section{Conclusion}

Using an ultra-high vacuum furnace, aluminum was purified to 6-nine level, and this purification process succeeded in saving time in comparison with the zone refining method. Furthermore, a fairly large single crystal of several centimeters in dimension was obtained by the present method, and the dislocation density decreased to $10^{7} \mathrm{~m}^{-2}$ by cyclic annealing. It was found that elements with high purification efficiency have a distribution coefficient of less than one unit.

It is expected that these nearly perfect single crystals contribute to the advanced research in metal physics. 
Table 4 The residual resistivity estimated from chemical composition using the specific electrical resistivity.

\begin{tabular}{|c|c|c|c|c|c|c|}
\hline \multirow{2}{*}{ Element } & \multicolumn{2}{|c|}{$\begin{array}{c}\text { Chemical composition, } \\
\text { C/at-ppm }\end{array}$} & \multirow{2}{*}{$\begin{array}{c}\text { Specific } \\
\text { resistivity, } \\
\text { S.R } \\
/ \mathrm{p} \Omega \cdot \mathrm{m} \mathrm{ppm}^{-1}\end{array}$} & \multirow{2}{*}{ Ref. } & \multicolumn{2}{|c|}{$\begin{array}{l}\text { Estimated resistivity, } \\
\qquad \rho / \mathrm{p} \Omega \cdot \mathrm{m}\end{array}$} \\
\hline & Starting material & After melting & & & Starting material & After melting \\
\hline B & 0.27 & 0.025 & $1 ?$ & - & 0.27 & 0.02 \\
\hline $\mathrm{Mg}$ & 1.0 & 0.001 & 0.39 & 8) & 0.39 & 0.00 \\
\hline $\mathrm{Si}$ & 1.7 & 0.71 & 0.65 & 8) & 1.1 & 0.46 \\
\hline $\mathrm{Ti}$ & 0.12 & 0.12 & 5.5 & 6) & 0.68 & 0.65 \\
\hline $\mathrm{V}$ & 0.058 & 0.058 & 7 & 7) & 0.41 & 0.41 \\
\hline $\mathrm{Cr}$ & 0.031 & 0.030 & 8 & 7) & 0.25 & 0.24 \\
\hline Mn & 0.003 & 0.003 & 6.5 & 6) & 0.02 & 0.02 \\
\hline $\mathrm{Fe}$ & 0.30 & 0.072 & 6.5 & 7) & 1.9 & 0.47 \\
\hline $\mathrm{Ni}$ & 0.19 & 0.010 & 0.1 & 6) & 0.02 & 0.00 \\
\hline $\mathrm{Cu}$ & 0.27 & 0.19 & 0.75 & 6) & 0.20 & 0.14 \\
\hline $\mathrm{Zr}$ & 0.001 & 0.001 & 4.5 & 6) & 0.01 & 0.01 \\
\hline $\mathrm{Sb}$ & 0.000 & 0.000 & 1 & 7) & 0.00 & 0.00 \\
\hline $\mathrm{La}$ & 0.033 & 0.000 & $?$ & & 0.00 & 0.00 \\
\hline $\mathrm{Ce}$ & 0.087 & 0.000 & $?$ & & 0.00 & 0.00 \\
\hline $\mathrm{Nd}$ & 0.016 & 0.000 & $?$ & & 0.00 & 0.00 \\
\hline Th & 0.006 & 0.000 & $?$ & & 0.00 & 0.00 \\
\hline $\mathrm{U}$ & 0.005 & 0.000 & $?$ & & 0.00 & 0.00 \\
\hline Total & 4.133 & 1.223 & & & 5.31 & 2.42 \\
\hline$R R R$ & & & & & $5.1 \times 10^{3}$ & $1.13 \times 10^{4}$ \\
\hline
\end{tabular}

Table 5 The purification efficiency, melting point, vapor pressure at $660^{\circ} \mathrm{C}$ and distribution coefficient for impurity elements.

\begin{tabular}{|c|c|c|c|c|}
\hline Element & $\begin{array}{l}\text { Purifiction } \\
\text { efficiency } \\
\text { P.E. }\end{array}$ & $\begin{array}{c}\text { Melting point, } \\
\text { M.P. } /{ }^{\circ} \mathrm{C}\end{array}$ & $\begin{array}{c}\text { Vapor pressure } \\
\text { at } 660^{\circ} \mathrm{C} \\
\text { V.P./Pa }\end{array}$ & $\begin{array}{c}\text { Distribution } \\
\text { coeffcient } \\
\text { D.C. }\end{array}$ \\
\hline B & 0.9 & 2030 & $<1 \times 10^{-8}$ & $>1$ \\
\hline $\mathrm{Mg}$ & 1.0 & 650 & $7 \times 10^{2}$ & 0.51 \\
\hline $\mathrm{Si}$ & 0.6 & 1415 & $<1 \times 10^{-8}$ & 0.14 \\
\hline $\mathrm{Ti}$ & 0.0 & 1820 & $<1 \times 10^{-8}$ & 2.1 \\
\hline $\mathrm{V}$ & 0.0 & 1735 & $<1 \times 10^{-8}$ & 3.3 \\
\hline $\mathrm{Cr}$ & 0.0 & 1800 & $<1 \times 10^{-8}$ & 1.7 \\
\hline Mn & 0.0 & 1260 & $1 \times 10^{-3}$ & 0.7 \\
\hline $\mathrm{Fe}$ & 0.8 & 1530 & $<1 \times 10^{-8}$ & 0.03 \\
\hline $\mathrm{Ni}$ & 0.9 & 1455 & $<1 \times 10^{-8}$ & 0.008 \\
\hline $\mathrm{Cu}$ & 0.3 & 1080 & $1 \times 10^{-7}$ & 0.14 \\
\hline $\mathrm{Zn}$ & 1.0 & 419 & $6 \times 10^{3}$ & 0.75 \\
\hline $\mathrm{Zr}$ & 0.0 & 1750 & $<1 \times 10^{-8}$ & 2.6 \\
\hline $\mathrm{Sb}$ & 0.0 & 630 & $5 \times 10$ & $?$ \\
\hline $\mathrm{La}$ & 1.0 & 920 & $<1 \times 10^{-8}$ & 0.4 \\
\hline $\mathrm{Ce}$ & 1.0 & 800 & $<1 \times 10^{-8}$ & $<1$ \\
\hline $\mathrm{Nd}$ & 1.0 & 840 & $1 \times 10^{-7}$ & $?$ \\
\hline $\mathrm{Th}$ & 1.0 & 1840 & $<1 \times 10^{-8}$ & 0.06 \\
\hline $\mathrm{U}$ & 1.0 & 1130 & $<1 \times 10^{-8}$ & $<1$ \\
\hline $\mathrm{Al}$ & & 660 & $1 \times 10^{-6}$ & \\
\hline
\end{tabular}

\section{Acknowledgements}

The authors express their sincere thanks to Sumitomo Chemical Co. Ltd. for the supply of aluminum samples and for the analysis of vacuum-melted aluminum by GDMS. The authors wish to thank to Mr. K. Fujii of the technical staff of the Research Institute of Advanced Technology of Hiroshima Kokusai Gakuin University for his assistance in this work. This work is supported by a Grant-in-Aid for Scientific Research from the Ministry of Education, Culture, Sports, Science and Technology.

\section{REFERENCES}

1) E. Hashimoto and Y. Ueda: Mater. Trans., JIM 35 (1994) 262-265.

2) E. Hashimoto, Y. Ueda and T. Kino: supplément au J. de Physique III 5 (1995) C7-153-157.

3) Y. Ueda, K. Sakoh and E. Hashimoto: J. Phys. Soc. Jpn. 65 (1996) 3442-3444.

4) H. Osono, E. Hashimoto and T. Kino: Philos. Mag. 77 (1998) 1147-1160.

5) K. Matsusaka, H. Osono and T. Kino: Mater. Trans., JIM 41 (2000) 203-209.

6) K. Schröder: CRC Handbook of Electrical Resistivities of binary Metallic Alloys, (CRC Press, Boca Raton, 1983) pp. 25.

7) Japan Institute of Light Metals: Crystalline Structures and Properties of Aluminum, (JILM, Tokyo, 1991) pp. 159 (in Japanese).

8) S. Kawata and T. Kino: J. Phys. Soc. Jpn. 39 (1975) 684-691.

9) M. Hansen and K. Anderko: Constitution of Binary Alloys, (McGraw-Hill, New York, 1985). 